\title{
Integrating heart rate variability and electrochemical skin conductance as severity screening of cardiovascular autonomic neuropathy in type 2 diabetes
}

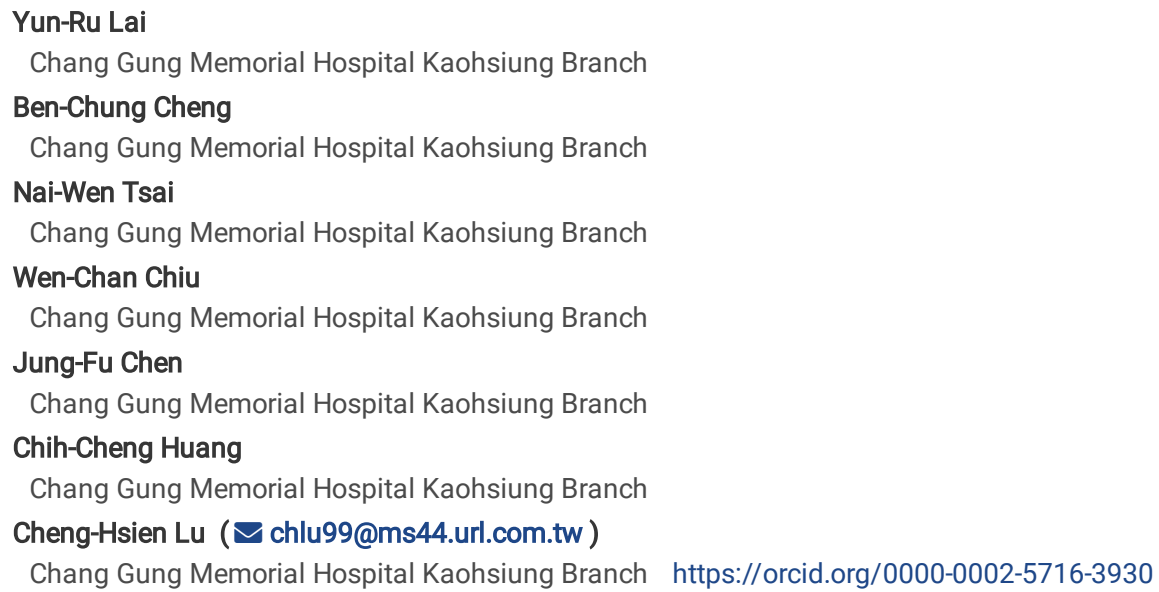




\section{Abstract}

Background: Clinical studies show that either heart rate variability (HRV) or electrochemical skin conductance (ESC) alone can serve as a simple and objective method for screening cardiovascular autonomic neuropathy (CAN). We tested the hypothesis that combining these two quantitative approaches can provide a better estimate of CAN severity in patients with type 2 diabetes (T2DM) who had already suffered from CAN in outpatient clinics.

Methods: Each patient received a complete battery of cardiovascular autonomic reflex tests (CARTs), with ESC measured by SUDOSCAN, time domain measured by standard deviation of all normal RR intervals (SDNN) and frequency domain of HRV (low frequency [LF], high frequency [HF], and LF/HF ratio), and peripheral blood studies for vascular risk factors. Severity of CAN was measured by CAN score.

Results: The 90 T2DM patients included 50 males and 40 females. Those with more severe CAN had a higher CAN score value $(P<0.0001)$ and lower values in feet ESC $(P=0.023)$ and SDNN $(P<0.0001)$. Stepwise linear regression analysis also showed that feet $E S C$ and $S D N N$ value $(P<0.0001$ and $P<0.0001)$ were significantly associated with CAN score, respectively.

Conclusions: Based on our results, a combination of electrophysiologic biomarkers (SDNN and feet ESC) as a test battery can improve the diagnostic accuracy and reinforce the accuracy in estimating CAN severity and can serve as a time-effective screening service in outpatient clinics.

\section{Background}

Diabetic cardiovascular autonomic neuropathy (CAN), the impairment of the autonomic balance of the cardiovascular (CV) system in the setting of diabetes mellitus (DM), strongly influences various CV diseases, causes detrimental effects on the quality of life, and leads to severe morbidity and mortality in patients with type 1 and type 2 diabetes mellitus (T1DM and T2DM) [1, 2]. The autonomic nervous system is one of the major homeostatic regulatory systems of the body. Sympathovagal balance failure with a sympathetic dominance is the main trigger of lethal arrhythmias and sudden death.

The prevalence of CAN is variable and depends on the definition and criteria used for diagnosis. A joint consensus statement by the American Diabetes Association (ADA) and American Academy of Neurology (AAN) has recommended that a battery of cardiovascular autonomic reflex tests (CARTs) should be performed to assess CAN [3].

Reduced heart rate variability (HRV) is an independent adverse prognostic factor used to detect CAN. HRV represents one of the most promising markers. A joint European Society of Cardiology and North American Society of Pacing and Electrophysiology task force defined and established standards of measurement, physiological interpretation, and clinical use of HRV, and the most widely used methods can be grouped under the time domain and frequency domain methods [4,5]. Established clinical data based on numerous studies consider HRV have been proven useful in detecting CAN in diabetes patients [6, 7]. Recently, portable devices that measure HRV provide cost-benefit along with the simplicity of the measurement and increased compliance for both research and clinical studies [8].

Sudomotor dysfunction, a length-dependent pattern of diseases in thin, non-myelinated sympathetic C-fibers, has been observed in both prediabetic and diabetic patients. The consensus statement of the ADA, AAN, and Latin American Diabetes Association includes assessing the sudomotor function's role in the early diagnosis of CAN in patients with diabetes $[9,10]$. Sudomotor function can be assessed using SUDOSCAN (Impeto Medical, Paris, France), which measures electrochemical skin conductance (ESC), which is a technological advance that calculates sweat function through the quantization of chronoamperometry measures of the hands and feet. It was recently developed to allow the measurement of diabetic small-fiber neuropathy and autonomic dysfunction [11].

Although HRV and ESC are validated and can serve as screening tools for CAN in patients with diabetes [5, 12-16], there is paucity of information that focuses on its role in estimating CAN severity in patients with diabetes who was already diagnosed with CAN in outpatient clinics, owing to the possible benefits of exploring the role of the $\mathrm{CV}$ autonomic function on subsequent $\mathrm{CV}$ events and the consequent development of therapeutic strategies to reduce the prevalence of CV events.

In this study, we evaluated the feasibility of a time-effective CV autonomic screening service at the outpatient clinic, including the time and frequency domains of HRV as well as the ESC. First, we evaluated which parameters in both HRV and ESC can serve as diagnostic biomarkers in the presence of CAN. Second, we combined the biomarkers in a test battery for CAN severity screening. We tested the hypothesis that CAN score and electrophysiologic parameters (time and frequency domains of HRV and ESC) have a strong association. If our hypothesis is true, when combined as a test battery, these two quantitative approaches can provide a better estimate of CAN severity and improve examinations.

\section{Patients And Methods}

\section{Study population}

A total of 90 patients ( $\geq 20$ years old) with T2DM who visited the outpatient diabetic clinic at Kaohsiung Chang Gung Memorial Hospital in Taiwan were included. Exclusion criteria included those who (1) suffered from moderate-to-severe heart failure (New York Heart Association class III and IV) and (2) had any type of arrhythmia that prevents the analysis of HRV, or pacemaker implantation due to any cause. This study was approved by the Ethics Committee of Chang Gung Memorial Hospital Institutional Review Board (201800388B0C501 and 201901363B0).

\section{Baseline clinical and laboratory measurements}


All patients underwent complete neurological and physical examinations upon enrollment and at their subsequent follow-ups at the outpatient clinic. A detailed medical history regarding prior use of medications was obtained from the patients and their families through standardized questions. Demographic data, including age, sex, duration of diabetes (years), body mass index (BMI), systolic and diastolic blood pressure (SBP and DSP), waist circumference (WC) during autonomic function testing, underlying disease (hypertension, coronary artery disease [CAD], ischemic stroke, and diabetic retinopathy [DR]), and laboratory parameters, were obtained at baseline.

\section{Biochemical analysis}

Blood samples were obtained by antecubital vein puncture in a fasting, nonsedative state between 09:00 and 10:00 AM in the control and study groups to exclude the possible influence of circadian variations. All blood samples were collected in Vacutainer SST tubes (BD, Franklin Lakes, NJ) and centrifuged at $3000 \mathrm{rpm}$ for $10 \mathrm{~min}$, and serum samples were collected and stored at $-80^{\circ} \mathrm{C}$ in multiple aliquots prior to biochemical measurement.

Serum levels of triglycerides, total cholesterol, high-density lipoprotein cholesterol (HDL-C), low-density lipoprotein cholesterol (LDL-C), blood sugar, HBA1C, and high-sensitivity C-reactive protein (hs-CRP) were analyzed by the hospital's central laboratory. Estimated glomerular filtration rate (eGFR) in each patient was calculated using an equation for Chinese subjects, as previously described [17]. Urine albumin/creatinine ratio (UACR) is less than $30 \mathrm{mg} / \mathrm{day}$; therefore, UACR between 30 and $300 \mathrm{mg} /$ day is called microalbuminuria, and UACR above $300 \mathrm{mg} /$ day is considered macroalbuminuria [18].

\section{Assessment of electrochemical skin conductance and SUDOSCAN risk scores}

ESC were assessed with patients placing their hands and feet on electrode plates for 3 min [19]. Measurement was based on an electrochemical reaction between electrodes and chloride ions, after stimulation of small fibers innervating the sweat glands with a low-voltage current ( $<4 \mathrm{~V})$. An ESC measurement for the hands and feet was generated from the derivative current associated with the applied voltage. Lower ESC values indicated dysfunction of the sweat glands.

SUDOSCAN risk scores were calculated automatically from ESC values, BMI, and age using an algorithm included in the device software. Scores were presented as percentages. Higher SUDOSCAN risk scores have been related to increased risk of cardiac autonomic abnormalities. Both ESC values and SUDOSCAN risk scores were displayed numerically with graphs on the device monitor [12].

\section{Assessment and scoring of cardiovascular autonomic functions}

CARTs are considered as gold-standard measures of autonomic function in patients with diabetes [3]. Parameters that were computed using Ewing's methods, including heart rate responses to deep breathing (E:I ratio), standing (30:15 ratio), and Valsalva maneuver and blood pressure responses to standing [20], were often used by diabetologists. These autonomic parameters were also obtained, and CAN was defined as the presence of at least two abnormal test results [3].

The severity of CAN was quantitated by summation of points obtained from each of the four tests, where each test was given a point of 0 or 1 if it yielded normal or abnormal values, respectively. We termed this severity score as CAN score. Therefore, CAN score provided a score from 0 to 4 points in this study. In this study, CAN is defined as no CAN, early CAN, definite CAN, and severe CAN if no, one, two, and more than two abnormal autonomic function testings.

\section{Parameters of heart rate variability (HRV)}

Using the 5-min resting electrocardiogram (ECG) recording, the standard deviation of all normal RR intervals (SDNN) was calculated as the time domain parameter of HRV. In addition, power spectral density analysis of HRV was also done to obtain the frequency domain parameters. Three main spectral components were distinguished in a spectrum calculated from the 5-min recording, i.e., high frequency (HF, $0.15-0.4 \mathrm{~Hz})$, low frequency (LF, $0.04-0.15 \mathrm{~Hz})$, and very low frequency (VLF, $0-0.04 \mathrm{~Hz}$ ). The components of LF and HF were computed both in absolute values of power (ms ${ }^{2}$ ) and in normalized unit (n.u.). The LF/HF ratio, regarded as an index of sympathovagal balance, was also calculated [16]. The aforementioned computing process was done by Kubios HRV Standard version 3.2 (Kubios Oy, Finland)

\section{Statistical analysis}

Data are expressed as means \pm standard deviations (SDs) or medians (interquartile ranges (IQR)). Categorical variables were compared using chi-square or Fisher's exact tests. Continuous variables that were not normally distributed by Kolmogorov-Smirnov test were logarithmically transformed to improve normality and compared. Five separate statistical analyses were performed. First, patients were stratified into four groups according to the severity of CAN. Second, receiver operating characteristic (ROC) curves were generated for parameters of ESC and time and frequency of HRV and CARTs in the presence of CAN. The area under the ROC curve (AUC) for the presence of CAN in addition to the sensitivity, specificity, and Youden's index of each parameter was also calculated. Fourth, correlation analysis was used to evaluate the relationship between the CAN score and the variables that included age, diabetes duration, $\mathrm{BMI}$, and parameters of ESC and time and frequency of HRV. Finally, stepwise models of multiple linear regression analysis were used to evaluate the influence of independent variables on the mean CART score. All statistical analyses were conducted using the SAS software package, version 9.1 (2002, SAS Statistical Institute, Cary, North Carolina).

\section{Results}

\section{General characteristics of patients with diabetes stratified by severity of cardiovascular autonomic neuropathy}

The 90 patients with diabetes included 50 males and 40 females. Patient characteristics and baseline underlying diseases at assessment are presented in Table 1. 
Those with more severe CAN had higher UACR $(P=0.012)$. Those with more severe CAN had higher CART score value $(P<0.0001)$ and lower values in parasympathetic parameters, including the Valsalva ratio (VR), E:I ratio, and 30:15 ratio $(P<0.0001, P<0.0001$, and $P<0.0001$, respectively), and lower values in feet ESC $(\mu S)(P=0.023)$, BP change related to standing $(P<0.0001)$, and SDNN $(<0.0001)$. Those with more severe CAN had a higher prevalence of orthostatic hypotension $(\mathrm{P}<0.0001)$ (Table 2$)$.

\section{Diagnostic accuracy for parameters of autonomic functions in predicting cardiovascular autonomic neuropathy, using ROC curve analysis}

Statistical analyses for parameters of autonomic functions in predicting CAN using ROC curve analysis were as follows: $30: 15$ ratio $(P<0.0001)$, VR $(P<0.0001)$, E:I ratio $(P<0.0001)$, BP change related to standing $(P=0.005)$, SDNN $(P<0.0001)$, total spectral power of $L F(P<0.0001)$, total spectral power of $H F$ $(P<0.0001), L F$ (n.u.) $(P=0.722), \operatorname{HF}$ (n.u.) $(P=0.722), L F / H F$ ratio $(P=0.542)$, hand ESC $(\mu S)(P=0.145)$, feet ESC $(\mu S)(P=0.009), S U D 0 S C A N$ risk score $(P=0.063)$, and combined SDNN and feet ESC $(P<0.0001)$. Further, the sensitivity, specificity and AUC for statistically significant parameters of HRV and ESC in predicting CAN, using ROC curve analysis, are listed in Table 3 and Figure 1.

\section{Effect of autonomic function parameters on cardiovascular autonomic reflex test scores}

Correlation analysis parameters used to evaluate the effects of autonomic function parameters on CAN score are listed in Table 4. The significant statistical results (correlation coefficient, $P$ value) on CAN score were as follows: age (year) ( $r=-0.332, P=0.002)$, diabetes duration (year) ( $r=0.240, P=0.025)$, $S D N N$ ( $m s$ ) $(r=-0.462, P<0.0001), \operatorname{LF}$ (power density, ms2) $(r=-0.455, P<0.0001), \operatorname{HF}($ power density, ms2) $(r=-0.227, P=0.033)$, and feet $E S C(\mu S)(r=-0.313, P=0.003)$.

\section{Effects of the variables on cardiovascular autonomic reflex tests}

The effects of the variables on CAN score in patients with T2DM according to correlation analysis are listed in Table 4. Based on the correlation analysis, only significant variables were enrolled into stepwise linear regression models. Results from the model analysis (Table 5) revealed that only age, SDNN, and feet ESC value were significantly associated with CAN score and were enrolled into stepwise linear regression models. The formula is as follows: CAN score= 5.936 $-0.026 \times($ SDNN $)-0.045 \times($ age $)-0.019 \times($ feet ESC) .

\section{Discussion}

Clinical data show that clinical manifestations of CAN appear in T2DM, and the estimated 5-year mortality is approximately 50\% [21]. Thus, evaluating the severity of CAN regression or progression is important for risk stratification and subsequent management. To our knowledge, efforts on those patients who had already suffered from CAN remain unsatisfactory.

\section{Major findings of our study}

Our study produced three major findings. First, SDNN, total power of HF and LF, and feet ESC had sufficient diagnostic accuracy in the presence of CAN in ROC analysis though only either HRV or ESC as a screening tool could be unsatisfactory. However, combined SDNN and feet ESC can increase the diagnostic accuracy of CAN (sensitivity, specificity, and AUC). Second, SDNN, total power of HF and LF, and feet ESC had been shown to bear a significant negative correlation with CAN score, indicating that they are surrogate markers of CAN severity. Finally, our study results showed that combining SDNN, total power of HF and LF, and feet ESC can improve assessment in a multiple linear regression model (R-squared value is highest in model 3).

\section{Risk factors associated with the severity of CAN}

The pathophysiological mechanism of CAN development is multifactorial, and several studies reported the important role of CV risk factors, such as diabetes duration, degree of glycemic control, SBP, triglyceride levels, and BMI, in the development of CAN [22-25]. Further, there is enough evidence that CAN may precede DM [24]. A framework for screening and early multifactorial interventions (hyperglycemia, hypertension, dyslipidemia, and microalbuminuria) is the best prospect for preventing or halting CAN and its devastating sequelae and is the gold standard of diabetic care in T2DM [26] and T1DM [27]. Diabetes duration is an important risk factor for CAN. CAN is a length-dependent pattern of disease, and parasympathetic activity can be damaged in the early phase of CAN with sympathetic predominance. As the diseases progress, sympathetic denervation occurs in the late stage of CAN [28]. Our study also showed that all parasympathetic parameters (e.g., VR, E:I ratio, 30:15 ratio, and SDNN) were significantly lower in proportion to the severity of CAN.

\section{Feet ESC and SDNN as electrophysiologic markers for evaluation of CAN severity}

CARTs are the gold standard for the diagnosis of CAN. Nevertheless, there are some limitations to perform the whole test battery in clinical practice. It takes around $30 \mathrm{~min}$ to complete all the tests, and thus screening a large number of patients with diabetes may be impossible in some busy clinics. In addition, the tests sometimes may not be done due to patients' limitation, such as patients' underlying conditions (e.g., dementia or parkinsonism) and those who are unable to do deep breathing or Valsalva maneuver, or patients who are unable to stand. Therefore, a simplified effective method may be needed in busy outpatient clinics so that most patients can be screened or frequently followed up.

A clinical study showed that ESC reduction is proportional to the skin nerve fiber density [29] and can be used as a noninvasive measurement and is correlated with the gold standard measurement for the severity of small fiber neuropathy (e.g., skin biopsy with quantization of intra-epidermal nerve fiber density). Furthermore, ESC can be a clinically meaningful tool to measure severity and follow-up for progression and regression [30]. Although several studies have demonstrated that SUDOSCAN risk score is a good screening test for CAN [12,31], the diagnostic accuracy of CAN in our study is not established. The available information was calculated automatically from ESC values, BMI, and age using an algorithm included in the device software as we did not know the 
detailed algorithm of SUDOSCAN risk scores. However, several CV risk factors, such as diabetes duration, degree of glycemic control, SBP, triglyceride levels, and diseases of central or peripheral autonomic dysfunctions, could contribute to the severity of CAN, but may not be considered in the device software. Further, different ethnic groups also can affect the normal values of ESC that seemed to lower in Chinese populations [32]. However, other studies and our study showed that feet ESC is significantly correlated with the severity of CAN [12], which is compatible with the length-dependent pattern of diseases.

Sympathetic and parasympathetic stimuli directly influence heart rate and are responsible for the physiologic variation in HRV. HRV can be evaluated in the time and frequency domains [33]. Short-term HRV may be another time-saving tool that can be used in busy clinics. Only a 5-min resting recording of ECG is needed for computation and the requirement of patient's cooperation is decreased.

There are several different approaches of HRV in the time and frequency domains. Among these methods, the time domain parameter, SDNN, is the most intuitional with the simplest computation. Furthermore, the parameter is essential for almost all portable HRV devices. Since variance is mathematically equal to the total power of spectral analysis, SDNN reflects all the cyclic components responsible for the variability at the time of recording.

Although parameters in the frequency domain may provide information about parasympathetic and sympathetic modulations or balance in addition to cardiovagal function [34], it bears some pitfalls in the interpretation of results. Application of the such technique is critically dependent on the understanding of the underlying physiology, the mathematical analyses used, and the many confounders and possible technical artifacts. The measurement of VLF, LF, and HF power components is usually made in absolute values of power (power density, $\mathrm{ms}^{2}$ ). LF and HF may also be measured in normalized units (n.u.), which represent the relative value of each power component in proportion to the total power minus the VLF component. The representation of LF (n.u.) and HF (n.u.) emphasizes the control and balance between the sympathetic and parasympathetic systems and tends to minimize the effect of the changes on the values of LF and HF total power components. Nevertheless, normalized units should always be quoted with absolute values of the LF and HF power in order to describe completely the distribution of power in spectral components. Our study showed that the values of LF and HF total power decreased in proportion to the severity to CAN, and both LF and HF total power were negatively correlated with the CAN score. It implies that both parasympathetic and sympathetic impairments were more severe as the severity of CAN progressed. In view of our current data in HRV, SDNN has the highest correlation coefficient with the CAN score among these HRV parameters. We recommend using SDNN as an indicator for the severity of CAN.

Finally, our study confirmed that this combination of electrophysiologic biomarkers (SDNN and feet ESC) as a simplified test battery is a feasible timeeffective CV autonomic screening service for outpatient clinics and can be completed in less than 10 min. It also reduces clinic visits and provides objective and quantitative measures of CAN in those patients who already had CAN. Similar to the idea of a one-stop service for microvascular screening [35], our test battery can serve as a one-stop CAN screening service. If the test battery shows CAN progression, they will resort to the more sophisticated and specific, but ultimately more time-consuming complete autonomic function testings (e.g., CARTs).

\section{Study Limitations}

This study is a cross-sectional prospective study. Although we observed close relationship between SDNN and feet ESC and the severity of CAN in this observational study, the role of the test battery together with the combination of the two electrophysiologic biomarkers (SDNN and feet ESC) in a longitudinal study to evaluate the regression or progression of CAN is mandatory. Second, our study only included a Chinese population with type 2 diabetes. We did not included those patients who had pre-diabetes, non-diabetes or normal control.

\section{Conclusion}

Based on our results, a combination of electrophysiologic biomarkers (SDNN and feet ESC) as a test battery can improve the diagnostic accuracy and reinforce the accuracy by estimating the severity of CAN and can serve as a time-effective screening service in outpatient clinics.

\section{Abbreviations}

HRV, heart rate variability; ESC, electrochemical skin conductance; CAN, cardiovascular autonomic neuropathy; SDNN, standard deviation of all normal RR intervals; CARTs, cardiovascular autonomic reflex tests; LF, low frequency; HF, high frequency; CV, cardiovascular; DM, diabetes mellitus; T1DM and T2DM, type 1 and type 2 diabetes mellitus; ADA, American Diabetes Association; AAN, American Academy of Neurology; CARTs, cardiovascular autonomic reflex tests; BMI, body mass index; SBP and DSP, systolic and diastolic blood pressure; WC, waist circumference; CAD, coronary artery disease; HDL-C, high-density lipoprotein cholesterol; LDL-C, low-density lipoprotein cholesterol; hs-CRP, high-sensitivity C-reactive protein; eGFR, Estimated glomerular filtration rate; UACR, albumin/creatinine ratio; ECG, electrocardiogram; SDs, standard deviations; IQR, interquartile ranges; AUC, area under the ROC curve; HbA1c, glycohemoglobin; VR, Valsalva ratio

\section{Declarations}

\section{Authors' contributions}

YRL participated in the design of the study and drafted the manuscript. WCC, CCH, NWT, BCC, and JFC participated in the sequence alignment and clinical evaluation of patients. $\mathrm{CCH}$ performed the statistical analysis. $\mathrm{CHL}$ and $\mathrm{CCH}$ conceived the idea for the study and participated in its design and coordination and helped draft the manuscript. All authors read and approved the final manuscript.

\section{Acknowledgements}

The authors thank all the subjects who participated in this study. 


\section{Competing interests}

The authors declare that they have no competing interests.

\section{Availability of data and materials}

The data from this study can be acquired from the corresponding author upon reasonable request.

\section{Consent for publication}

Not applicable.

\section{Ethical approval and consent to participate}

This study conformed to the guidelines of the Declaration of Helsinki and has been approved by the Institutional Review Board of Chang Gung Memorial Hospital (201800388B0C501 and 201901363B0).

\section{Funding}

This work was supported by grants from the Chang Gung Memorial Hospital (Chang Gung Medical Research Project CMRPG8H0501 and CMRPG8J1211).

\section{References}

1. Vinik Al, Maser RE, Mitchell BD, Freeman R: Diabetic autonomic neuropathy. Diabetes care 2003, 26(5):1553-1579.

2. Vinik Al, Ziegler D: Diabetic cardiovascular autonomic neuropathy. Circulation 2007, 115(3):387-397.

3. Spallone V, Bellavere F, Scionti L, Maule S, Quadri R, Bax G, Melga P, Viviani G, Esposito K, Morganti R: Recommendations for the use of cardiovascular tests in diagnosing diabetic autonomic neuropathy. Nutr Metab Cardiovasc Dis 2011, 21(1):69-78.

4. Malik M: Heart rate variability: Standards of measurement, physiological interpretation, and clinical use: Task force of the European Society of Cardiology and the North American Society for Pacing and Electrophysiology. Ann Noninvasive Electrocardiol. 1996, 1(2):151-181.

5. Cha SA, Park YM, Yun JS, Lee SH, Ahn YB, Kim SR, Ko SH: Time-and frequency-domain measures of heart rate variability predict cardiovascular outcome in patients with type 2 diabetes. Diabetes Res Clin Pract. 2018, 143:159-169.

6. Malpas SC, Maling TJ: Heart-rate variability and cardiac autonomic function in diabetes. Diabetes 1990, 39(10):1177-1181.

7. Bellavere F, Balzani I, De Masi G, Carraro M, Carenza P, Cobelli C, Thomaseth K: Power spectral analysis of heart-rate variations improves assessment of diabetic cardiac autonomic neuropathy. Diabetes 1992, 41(5):633-640.

8. Dobbs WC, Fedewa MV, MacDonald HV, Holmes CJ, Cicone ZS, Plews DJ, Esco MR: The accuracy of acquiring heart rate variability from portable devices: a systematic review and meta-analysis. Sports Med. 2019, 49(3):417-435.

9. Asbury A, Porte D: American Diabetes Association and American Academy of Neurology. Report and recommendations of the San Antonio conference on diabetic neuropathy (Consensus Statement). Diabetes Care 1988, 11(7):592.

10. Tesfaye S, Boulton AJ, Dyck PJ, Freeman R, Horowitz M, Kempler P, Lauria G, Malik RA, Spallone V, Vinik A: Diabetic neuropathies: update on definitions, diagnostic criteria, estimation of severity, and treatments. Diabetes care 2010, 33(10):2285-2293.

11. Casellini CM, Parson HK, Richardson MS, Nevoret ML, Vinik Al: Sudoscan, a noninvasive tool for detecting diabetic small fiber neuropathy and autonomic dysfunction. Diabetes Technol Ther. 2013, 15(11):948-953.

12. Yajnik C, Kantikar V, Pande A, Deslypere J-P, Dupin J, Calvet J-H, Bauduceau B: Screening of cardiovascular autonomic neuropathy in patients with diabetes using non-invasive quick and simple assessment of sudomotor function. Diabetes Metab. 2013, 39(2):126-131.

13. Yuan T, Li J, Fu Y, Xu T, Li J, Wang X, Zhou Y, Dong Y, Zhao W: A cardiac risk score based on sudomotor function to evaluate cardiovascular autonomic neuropathy in asymptomatic Chinese patients with diabetes mellitus. PLoS One 2018, 13(10).

14. Zhu L, Zhao X, Zeng P, Zhu J, Yang S, Liu A, Song Y: Study on autonomic dysfunction and metabolic syndrome in Chinese patients. J Diabetes Investig. 2016, 7(6):901-907.

15. Zeng Q, Dong S, Wang M, Wang F, Li J, Zhao X: Cardiac autonomic neuropathy risk estimated by sudomotor function and arterial stiffness in Chinese subjects. J Hum Hypertens. 2016, 30(11):720-725.

16. Electrophysiology TFotESoCtNASoP: Heart rate variability: standards of measurement, physiological interpretation, and clinical use. Circulation 1996, 93(5):1043-1065.

17. Ma YC, Zuo L, Chen JH, Luo Q, Yu XQ, Li Y, Xu JS, Huang SM, Wang LN, Huang W: Modified glomerular filtration rate estimating equation for Chinese patients with chronic kidney disease. J Am Soc Nephrol 2006, 17(10):2937-2944.

18. Chiu WC, Lai YR, Cheng BC, Huang CC, Chen JF, Lu CH: HbA1C Variability Is Strongly Associated with Development of Macroalbuminuria in Normal or Microalbuminuria in Patients with Type 2 Diabetes Mellitus: A Six-Year Follow-Up Study. Biomed Res Int. 2020, 2020.

19. Mayaudon H, Miloche P-O, Bauduceau B: A new simple method for assessing sudomotor function: relevance in type 2 diabetes. Diabetes Metab. 2010, 36(6):450-454.

20. Ewing D, Clarke B: Diagnosis and management of diabetic autonomic neuropathy. Br Med J (Clin Res Ed) 1982, $285(6346): 916$.

21. Ewing D, Campbell I, Clarke B: The natural history of diabetic autonomic neuropathy. QJM 1980, 49(1):95-108. 
22. Rolim LCdSP, Sá JRd, Chacra AR, Dib SA: Diabetic cardiovascular autonomic neuropathy: risk factors, clinical impact and early diagnosis. Arq Bras Cardiol. 2008, 90(4):e24-e32.

23. Dafaalla MD, Nimir MN, Mohammed MI, Ali OA, Hussein A: Risk factors of diabetic cardiac autonomic neuropathy in patients with type 1 diabetes mellitus: a meta-analysis. Open heart 2016, 3(2):e000336.

24. Valensi P, Paries J, Attali J, for Research FG: Cardiac autonomic neuropathy in diabetic patients: influence of diabetes duration, obesity, and microangiopathic complications-the French multicenter study. Metabolism 2003, 52(7):815-820.

25. Lai YR, Huang CC, Chiu WC, Liu RT, Tsai NW, Wang HC, Lin WC, Cheng BC, Su YJ, Su CM, Hsiao SY, Wang PW, Chen JF, Lu CH: HbA1C variability is strongly associated with the severity of cardiovascular autonomic neuropathy in patients with Type 2 diabetes after longer diabetes duration. Front Neurosci. 2019, 13:458.

26. Gæde P, Vedel P, Parving H-H, Pedersen O: Intensified multifactorial intervention in patients with type 2 diabetes mellitus and microalbuminuria: the Steno type 2 randomised study. Lancet. 1999, 353(9153):617-622.

27. The effect of intensive diabetes therapy on measures of autonomic nervous system function in the Diabetes Control and Complications Trial (DCCT). Diabetologia 1998, 41(4):416-423.

28. Ewing DJ, Martyn CN, Young RJ, Clarke BF: The value of cardiovascular autonomic function tests: 10 years experience in diabetes. Diabetes care 1985, 8(5):491-498.

29. Novak P: Electrochemical skin conductance correlates with skin nerve fiber density. Front Aging Neurosci. 2016, 8:199.

30. Vinik Al, Nevoret ML, Casellini C: The new age of sudomotor function testing: a sensitive and specific biomarker for diagnosis, estimation of severity, monitoring progression, and regression in response to intervention. Front Endocrinol.. 2015, 6:94.

31. Selvarajah D, Cash T, Davies J, Sankar A, Rao G, Grieg M, Pallai S, Gandhi R, Wilkinson ID, Tesfaye S: SUDOSCAN: a simple, rapid, and objective method with potential for screening for diabetic peripheral neuropathy. PLoS One 2015, 10(10).

32. Vinik Al, Smith AG, Singleton JR, Callaghan B, Freedman BI, Tuomilehto J, Bordier L, Bauduceau B, Roche F: Normative values for electrochemical skin conductances and impact of ethnicity on quantitative assessment of sudomotor function. Diabetes Technol Ther. 2016, 18(6):391-398.

33. Bernardi L, Spallone V, Stevens M, Hilsted J, Frontoni S, Pop-Busui R, Ziegler D, Kempler P, Freeman R, Low P: Methods of investigation for cardiac autonomic dysfunction in human research studies. Diabetes Metab Res Rev. 2011, 27(7):654-664.

34. Malliani A, Pagani M, Montano N, Mela GS: Sympathovagal balance: a reappraisal. Circulation 1998.

35. Binns-Hall O, Selvarajah D, Sanger D, Walker J, Scott A, Tesfaye S: One-stop microvascular screening service: an effective model for the early detection of diabetic peripheral neuropathy and the high-risk foot. Diabet Med. 2018, 35(7):887-894.

\section{Tables}

\section{Table 1. Baseline characteristics of patients with Type 2 diabetes}


Characteristics

Age (year)

Sex (male/female)

Diabetes duration (year)

Body mass index

Waist circumference $(\mathrm{cm})$

$S B P(\mathrm{mmHg})$

$D B P(\mathrm{mmHg})$

Baseline underlying disease

Hypertension (\%)

Coronary heart disease (\%)

Cerebrovascular events (\%)

Hyperlipidemia (\%)

Chronic kidney diseases (\%)

Retinopathy, n (\%)

Proteinuria, $n(\%)$

Other concomitant medications

$A C E$ inhibitor or $A R B$ (\%)

Beta-blocker (\%)

Calcium channel blocker (\%)

Diuretics (\%)

Antiplatelet medications (\%)

Statins (\%)

Abbreviations: $n$, number of cases; IQR= interquartile range, SBP, systolic blood pressure; DBP, diastolic blood pressure; OHA, oral hypoglycemic agent; $A C E$, angiotensin-converting enzyme; $A R B$, angiotensin II receptor blocker

Table 2. Laboratory and autonomic function testings in patients with Type 2 diabetes stratified by the severity of cardiovascular autonomic neuropathy
$67.5 \pm 8.1$

$50 / 40$

$11.5 \pm 8.6$

$27.1 \pm 4.6$

$90.6 \pm 11.7$

$137.8 \pm 17.9$

$76.5 \pm 12.4$

$67(74.4 \%)$

11 (12.2\%)

32 (35.6\%)

$70(77.8 \%)$

47 (52.2\%)

50 (55.6\%)

37 (41.1\%)

$64(71.1 \%)$

27 (30\%)

45 (50\%)

18 (20\%)

$26(28.8 \%)$

$69(76.7 \%)$ 


$\begin{array}{ll}\text { No } C A N & \text { Early CAN }\end{array}$

Laboratory test findings

\begin{tabular}{|c|c|c|c|}
\hline Total cholesterol $(\mathrm{mmol} / \mathrm{L})$ & $158.0 \pm 35.5$ & $152.8 \pm 36.5$ & 15 \\
\hline Triglyceride $(\mathrm{mmol} / \mathrm{L})$ & $109.8 \pm 51.5$ & $145.5 \pm 82.6$ & 14 \\
\hline$H D L-C(\mathrm{mmol} / \mathrm{L})$ & $47.1 \pm 9.2$ & $45.4 \pm 12.1$ & $4 \longleftarrow$ \\
\hline$L D L-C(\mathrm{mmol} L)$ & $88.9 \pm 31.0$ & $82.9 \pm 22.3$ & 86 \\
\hline $\mathrm{HbA7c}$ & $6.8 \pm 0.8$ & $6.8 \pm 0.9$ & 7.1 \\
\hline
\end{tabular}

(\%)

$6.9 \pm 0.9$

$7.4 \pm 0.9$

$7.3 \pm 1.1$

$7.4 \pm 1.3$

0.171

eGFR

$62.3 \pm 22.1 \quad 60.0 \pm 28.1$

$U A C R$

$14.2 \quad 10.5(7.7,76.6) \quad 15$

Hs-CRP

$(8.8,47.3)$

$1.6 \pm 0.9 \quad 1.8 \pm 1.4 \quad 1$.

CAN score

$\begin{array}{lll}0 & 1.0 \pm 0 & 2.1\end{array}$

E:I ratio

$30 / 15$ ratio

$\begin{array}{lll}1.2 \pm 0.1 & 1.1 \pm 0.1 & 1 .\end{array}$

Valsalva ratio

Orthostatic Hypotension

$\begin{array}{lll}1.2 \pm 0.1 & 1.1 \pm 0.04 & 1.1\end{array}$

BP change related to standing $(\mathrm{mmHg})$

$\begin{array}{lll}1.5 \pm 0.2 & 1.3 \pm 0.2 & 1.2\end{array}$

Heart rate variability

Time domain

$\operatorname{SDNN}(m s)$

$34.7 \pm 26.2 \quad 22.9 \pm 10.6 \quad$ 7\%

Frequency domain

LF (power density, $m s^{2}$ )

$\begin{array}{lll}0 & 0 & 0 \\ -3(-11,3) & -3(-8.5,3.5) & -5 . \\ & & -1 .\end{array}$

$L F($ n. u.)

HF (power density, $m s^{2}$ )

$H F$ (n. u.)

LF/HF ratio

\begin{tabular}{lll}
$\begin{array}{l}63.8(36.1, \\
203.3)\end{array}$ & $98.1(31.9,139.5)$ & $2 \xi$ \\
$7 \varepsilon$ \\
\hline $57.0 \pm 20.8$ & $45.8 \pm 22.8$ & $4 \epsilon$ \\
\hline $50.9(6.1$, & $101.1(35.9$, & 31 \\
$174.2)$ & $198.0)$ & 51 \\
$43.0 \pm 20.8$ & $54.2 \pm 22.8$ & $5 c$ \\
$1.7(0.7$, & $0.8(0.4,2.5)$ & 1.1 \\
$2.7)$ & & 2.1
\end{tabular}

Sudoscan

Hands ESC, $\mu S$

Feet $E S C, \mu S$

Sudoscan-risk scores (\%)

\begin{tabular}{lll}
$50.6 \pm 18.1$ & $48.8 \pm 23.0$ & 42 \\
\hline $59.4 \pm 16.1$ & $55.7 \pm 17.8$ & 44 \\
\hline $34.3 \pm 6.5$ & $36.7 \pm 8.9$ & $3 \varepsilon$
\end{tabular}

Data are presented as means \pm standard deviations or median (IQR) $n(\%) . *=P<0.05 ; * * P<0.01$

Abbreviations: IQR= interquartile range, CAN: cardiac autonomic neuropathy; $n$, number of cases; CARTs, cardiac autonomic reflex tests; ESC, electrochemical deviation of normal RR interval, $L F$, low frequency; $H F$, high frequency; n.u., normalized unit;

Table 3. Sensitivity, specificity, and area under the curve for parameters of cardiovagal functions in predicting cardiovascular autonomic neuropathy, using ROC curve analysis 


\begin{tabular}{|c|c|c|c|c|c|}
\hline Significant parameters & Cut-off value & $\operatorname{AUC}(95 \% \mathrm{Cl})$ & Sensitivity (\%) & Specificity (\%) & $P$-value \\
\hline \multicolumn{6}{|l|}{ CARTs } \\
\hline E:I ratio & 1.105 & $0.784(0.68-0.88)$ & 60.9 & 81.2 & $<0.0001 * *$ \\
\hline 30/15 ratio & 1.045 & $0.815(0.72-0.92)$ & 82.6 & 75 & $<0.0001 * *$ \\
\hline Valsalva ratio & 1.225 & $0.792(0.70-0.89)$ & 65.2 & 84.4 & $<0.0001^{* *}$ \\
\hline$B P$ change related to standing $(\mathrm{mmHg})$ & -8.5 & $0.687(0.57-0.81)$ & 7.17 & 56.2 & $0.005^{\star *}$ \\
\hline \multicolumn{6}{|l|}{ Heart rate variability } \\
\hline$S D N N(m s)$ & 16.45 & $0.737(0.63-0.85)$ & 76.1 & 56.2 & $<0.0001^{* *}$ \\
\hline$L F\left(\right.$ power density, $m s^{2}$ ) & 32.16 & $0.773(0.67-0.88)$ & 80.4 & 68.7 & $<0.0001 * *$ \\
\hline$H F\left(\right.$ power density, $m s^{2}$ ) & 44.9 & $0.751(0.52-0.70)$ & 73.9 & 71.9 & $<0.0001^{* *}$ \\
\hline \multicolumn{6}{|l|}{ Sudoscan } \\
\hline Feet $E S C, \mu S$ & 44.5 & $0.674(0.56-0.93)$ & 82.6 & 40.6 & $0.009 * *$ \\
\hline Combined SDNN and Feet ESC & 0.372 & $0.779(0.68-0.88)$ & 79.4 & 70 & $<0.0001^{* *}$ \\
\hline
\end{tabular}

$*=P<0.05 ; * * P<0.01 ;$ Abbreviation: ROC, receiver operating characteristic; CARTs, cardiac autonomic reflex tests; ESC, electrochemical skin conductance; SDNN standard deviation of normal RR interval, LF, low frequency; HF, high frequency;

Table 4. Correlation analysis of cardiac autonomic reflex tests in patients with type 2 diabetes

\begin{tabular}{|c|c|c|}
\hline \multirow[t]{2}{*}{ Variables } & \multicolumn{2}{|c|}{ CAN score } \\
\hline & $r$ & Pvalue \\
\hline Age (year) & -0.332 & $0.002^{* *}$ \\
\hline Diabetes duration (year) & 0.240 & $0.025^{*}$ \\
\hline Body mass index & 0.05 & 0.642 \\
\hline$S D N N(m s)$ & -0.462 & $<0.0001^{* *}$ \\
\hline$L F($ n. u. $)$ & -0.115 & 0.291 \\
\hline$H F($ n. u.) & 0.115 & 0.291 \\
\hline LF (power density, ms2) & -0.455 & $<0.0001^{* *}$ \\
\hline HF (power density, ms2) & -0.227 & $0.033^{*}$ \\
\hline$L F / H F$ ratio & -0.124 & 0.253 \\
\hline Hands ESC, $\mu S$ & -0.132 & 0.226 \\
\hline Feet ESC, $\mu S$ & -0.313 & $0.003^{* *}$ \\
\hline
\end{tabular}

$r$ : correlation coefficient. * indicates that $p$ value $<0.05$. ** indicates that $p$ value $<0.01$.

Abbreviations: $n$, number of cases; CARTs, cardiac autonomic reflex tests; ESC, electrochemical skin conductance; SDNN standard deviation of normal RR interval, $L F$, low frequency; $H F$, high frequency; n.u., normalized unit;

Table 5. Effects of the variables on composite autonomic scoring scale and cardiac autonomic reflex tests according to correlation analysis 
CAN score

\begin{tabular}{|c|c|c|c|c|c|c|}
\hline \multirow[t]{2}{*}{ variables } & \multicolumn{2}{|c|}{ Model 1} & \multicolumn{2}{|c|}{ Model 2} & \multicolumn{2}{|c|}{ Model 3} \\
\hline & Beta & $P$-value & Beta & $P$-value & Beta & $P$-value \\
\hline$S D N N(m s)$ & -0.026 & $<0.0001^{\star *}$ & -0.025 & $<0.0001^{\star *}$ & -0.026 & $<0.0001^{\text {** }}$ \\
\hline Age (year) & -0.327 & $0.001^{* *}$ & -0.042 & $0.001^{* *}$ & -0.045 & $<0.0001^{* *}$ \\
\hline Feet ESC, $\mu S$ & -0.304 & $0.002^{* *}$ & -0.328 & $<0.0001^{* *}$ & -0.019 & $<0.0001^{* *}$ \\
\hline Diabetes duration (year) & 0.124 & 0.229 & 0.142 & 0.14 & 0.025 & 0.799 \\
\hline LF (power density, ms2) & -0.055 & 0.591 & -0.101 & 0.3 & -0.022 & 0.812 \\
\hline HF (power density, ms2) & 0.055 & 0.591 & 0.101 & 0.3 & 0.022 & 0.812 \\
\hline
\end{tabular}

Regression coefficient for each individual variable. Abbreviations: CARTs: Cardiac autonomic reflex tests; ESC, electrochemical skin conductance; SDNN standard deviation of normal RR interval, $L F$, low frequency; $H F$, high frequency;

* indicates that $p$ value $<0.05$ ** indicates that $p$ value $<0.01$

a: Model summary

Model 1: Predictors: (constant), SDNN; $r^{2}=0.166$

Model 2: Predictors:(constant), SDNN, age, Feet ESC; $r^{2}=0.273$

Model 3: Predictors:(constant), SDNN, age, Feet ESC; $r^{2}=0.380$

Formula: CAN score=5.936-0.026 x (SDNN)-0.045x (Age) -0.019x (Feet ESC)

\section{Figures}

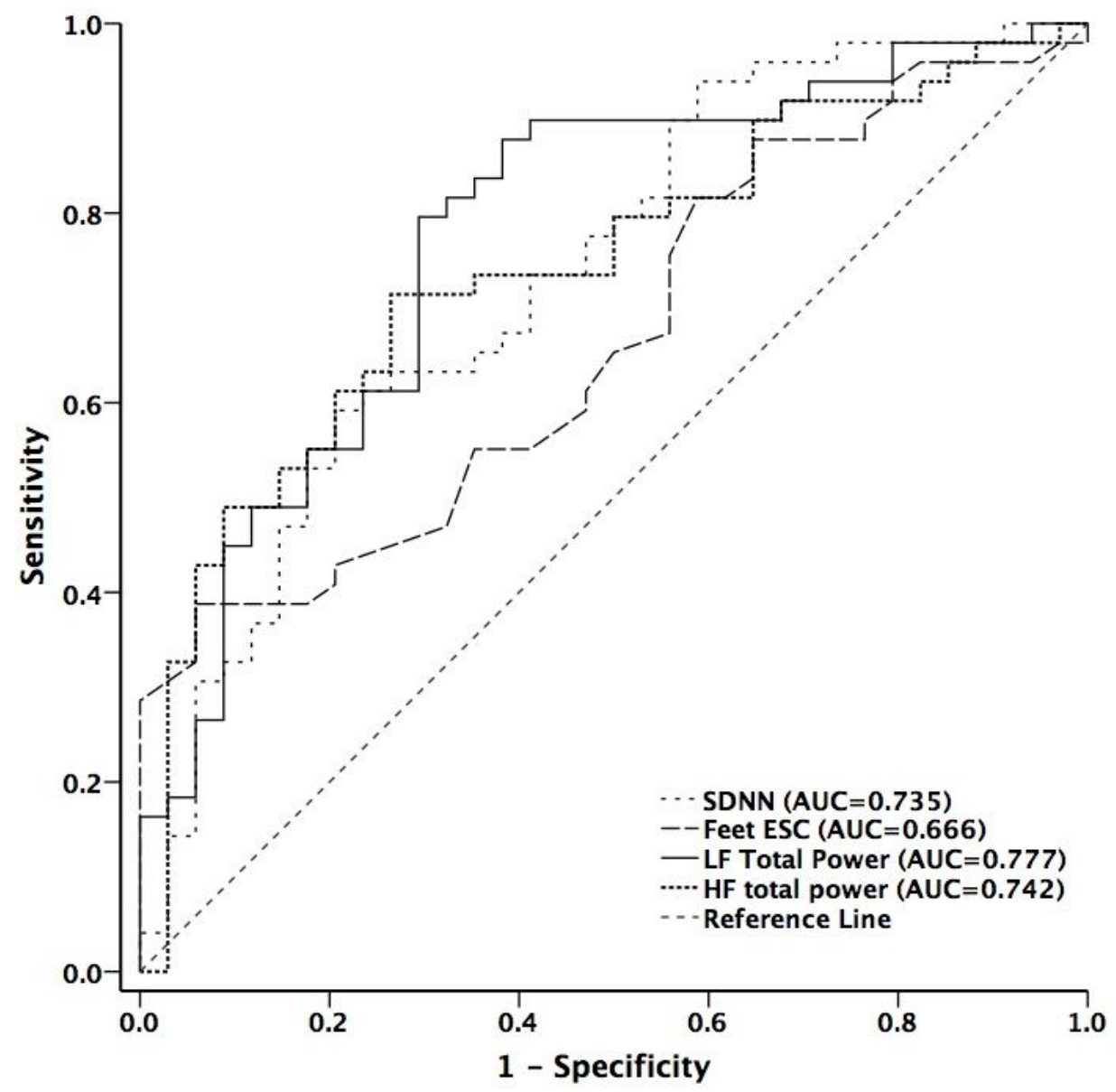

Figure 1 
Diagnostic accuracy and area under the curve for statistically significant parameters in predicting cardiovascular autonomic neuropathy, using ROC curve analysis
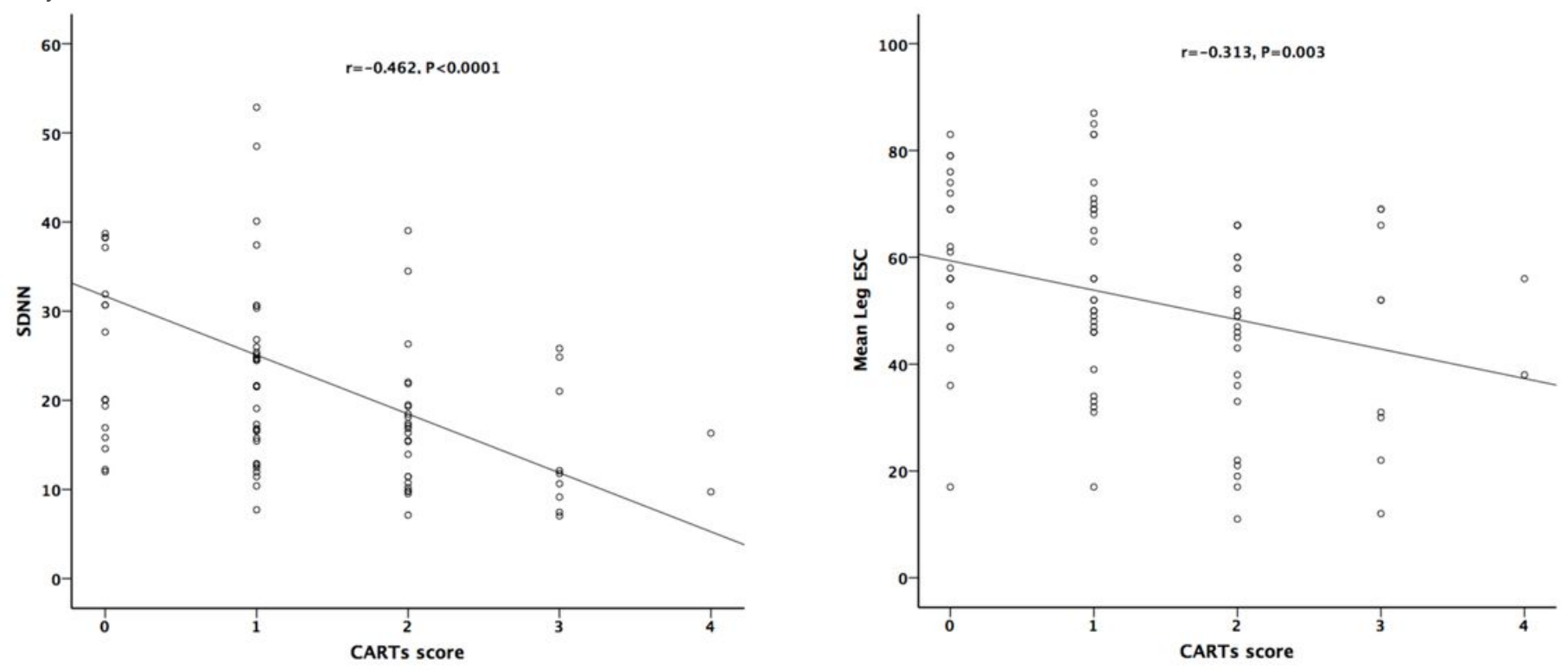

\section{Figure 2}

Relationship between SDNN value and CAN score (Figure 2 A) and relationship between Feet ESC value and CAN score (Figure 2 B) in patients with type 2 diabetes 